\title{
MS38-02 | Subpixel Spatial Resolution at 122 keV of an Spectroscopic Imager Composed of a pnCCD Coupled to a Columnar Csi(TL) SCIntillator
}

Schlosser, Dieter (PNSensor, Munich, GER); Hartmann, Robert (PNSensor, Munich, GER); Bechteler, Alois (PNSensor, Munich, GER); Abboud, Ali (University of Siegen, Siegen, GER); Shokr, Mohammad (University of Siegen, Siegen, GER); Pietsch, Ullrich (Univertity of Siegen, Munich, GER); Strüder, Lothar (PNSensor, Munich, GER)

New generation beam lines offer energies up to $150 \mathrm{keV}$ and above. With hard X-rays it is possible to investigate bulk properties of materials in Laue diffraction experiments with white X-ray beams. Therefore highly energy and spatially resolving detectors are required with high quantum efficiency up to $150 \mathrm{keV}$. We realized a detector consisting of a $450 \mu \mathrm{m}$ thick pnCCD with a pixel size of $75 \mu \mathrm{m} \times 75 \mu \mathrm{m}$ coupled to a $700 \mu \mathrm{m}$ thick columnar CsI(TI) scintillator with a high quantum efficiency in the energy range from $0.5 \mathrm{keV}$ to $100 \mathrm{keV}$ and above. The detector is front side illuminated, so that X-rays up to15 keV are mainly absorbed "directly" inside the pnCCD and energies above are mainly stopped in the scintillator, where the deposited energy is converted into scintillation photons, which are also detected by the pnCCD. This mode is called "indirect detection". In previous works we determined the energy resolution to be $\mathrm{FWHM} / \mathrm{E}=9 \%$ at $122 \mathrm{keV}$ for indirect detection and $0.7 \%$. In this work we have investigated the spatial resolution of the imaging detector in a "knife edge" experiment at $122 \mathrm{keV}$. We will show that the spatial resolution reaches subpixel values of about $30 \mu \mathrm{m}$, which is mainly limited by the track length of the generated secondaries inside the scintillator. This is in agreement with Geant4 Monte Carlo Simulations, which have been performed. 